\title{
ALGEBRAIC TOPOLOGY CRITERIA FOR MINIMAL SETS
}

\author{
HSIN $\mathrm{CHU}^{1}$
}

1. Introduction. Let $(X, T, \pi)$ be a transformation group, where $X$ is a compact Hausdorff space. If $T=R$, the additive group of all real numbers with the usual topology, this transformation group is called a continuous flow. If $T=Z$, the additive group of all integers with the discrete topology, this transformation group is called a discrete flow. A transformation group $(X, T, \pi)$ is called a minimal set if for each $x \in X$, the closure of the orbit of $x$ is $X$. (See $[2 ; 7 ; 12]$.) In this paper we prove the following result: "Let $(X, T, \pi)$ be a transformation group, where $X$ is a compact Hausdorff space and $T$ is a connected topological group or $T$ acts trivially on $H^{n}(X)$. If it is a minimal set with $\operatorname{cd}(X) \leqq n$ then $H^{n}(A)=0$ for every proper closed subset $A$ of $X$." Here $\operatorname{cd}(X)$ is the cohomological dimension of $X$ in the sense of $H$. Cohen (see $[3 ; 4]$ ). In this paper, the AlexanderSpanier cohomology group theory, with a fixed coefficient group $G$, is used. This result has a few consequences: for example, the universal curve of Menger and the universal curve of Sierpinski both can not be minimal under a connected group. This was an open question which was raised in an address (see [8]) by Professor W. H. Gottschalk. Using a similar approach we also prove the following result: "Let $(X, T, \pi)$ be a transformation group, where $X$ is a compact Hausdorff space and it contains more than one point and $T$ is connected. If $X$ is minimal and $H^{n}(X, D) \neq 0$ for some closed set $D$ in $X$, then it can not be disconnected by a closed subset of cohomology dimension $\leqq n-2$, , which is a generalization of a well-known theorem of $\mathrm{A}$. A. Markov (see [11]). This paper is also interesting in the use of Algebraic Topology in Topological Dynamics. The author wishes to take this opportunity to express his indebtedness to Professor W. H. Gottschalk and Professor H. C. Wang for their encouragement.

2. Theorems. It is known from [4] that for each $\zeta \in H_{n}(X, D)$, $\zeta \neq 0$ where $D$ is a closed subset of $X$, there exists a closed subset $A$ of $X$ such that $\zeta \mid(A, A \cap D) \neq 0$ and for all proper closed subsets $B$ of $A$ we have $\zeta \mid(B, B \cap D)=0$. We call this closed set $A$ a floor with respect to $\zeta$.

LEMMA 1. Let $(X, T, \pi)$ be a transformation group, where $X$ is a com-

Received by the editors June 12, 1961.

1 The author is indebted to the referee for his comments and suggestions. 
pact Hausdorff space with $\mathrm{cd}(X) \leqq n$ and $T$ is connected. If $H^{n}(X) \neq 0$ then for each $\zeta \in H^{n}(X), \zeta \neq 0$, each of its floors $A$ is an invariant closed set of $X$ under $T$.

Proof. We show $t A=A$ for every $t \in T$. Consider the commutative diagram:

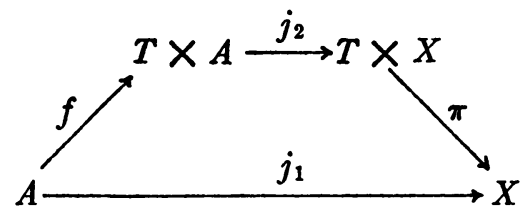

where $f(a)=(e, a)$, where $a \in A$ and $e$ is the identity of $T$, and $j_{1}$ and $j_{2}$ are the inclusion maps. Consider another commutative diagram:

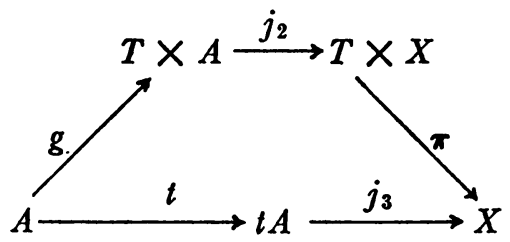

where $g(a)=(t, a)$ where $a \in A$ and a fixed $t \in T, j_{2}$ and $j_{3}$ are the inclusion maps and $t(a)=t a$. From the homotopy lemma (see [4]) we know $f^{*}=g^{*}$ on $H^{n}(T \times A)$ to $H^{n}(A)$. Consequently $j_{1}^{*}=t^{*} j_{3}^{*}$ on $H^{n}(X)$ to $H^{n}(A)$. It follows that the following diagram is commutative:

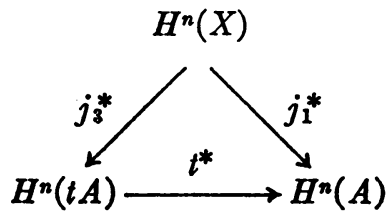

Thus $\beta \mid A \neq 0$ iff $\beta \mid t A \neq 0$ for every $\beta \in H^{n}(X)$. Since $H^{n}(X)$ $\rightarrow H^{n}(A \cup t A)$ is onto it follows that in the Mayer-Vietoris sequence

$$
\cdots \rightarrow B^{n}(A \cup t A) \stackrel{\phi}{\rightarrow} B^{n}(A) \times B^{n}(t A) \stackrel{\psi}{\rightarrow} B^{n}(A \cap t A) \rightarrow \cdots
$$

$H^{n}(A) \times\{0\}$ is mapped isomorphically by $\psi$ into $H^{n}(A \cap t A)$. Thus if $A$ is a floor for the element $\zeta \in H^{n}(X)$, then $A \cap t A=A$ for all $t \in T$ so that $A$ is invariant under $T$.

REMARK 1. We notice that the proof is still valid if one assumes only that $T$ acts trivially on $H^{n}(X)$ rather than that $T$ is connected.

THEOREM 1. Let $(X, T, \pi)$ be a transformation group, where $X$ is a 
compact Hausdorff space and $T$ is a connected topological group (or $T$ acts trivially on $\left.H^{n}(X)\right)$. If $(X, T, \pi)$ is a minimal set with $\operatorname{cd}(X) \leqq n$, then $H^{n}(B)=0$ for every proper, closed subset $B$ of $X$.

Proof. Suppose there is a closed subset $B$ of $X$ with the property that $H^{n}(B) \neq 0$, then, by the fact that $\operatorname{cd}(X) \leqq n$, we have $H^{n}(X) \neq 0$. For $\zeta \in H^{n}(X), \zeta \neq 0$, there exists a closed subset $A$ of $X$, which is a floor with respect to $\zeta$. By the preceding lemma (or Remark 1) we know $A$ is invariant under $T$. We know that $(X, T, \pi)$ is minimal if and only if whenever a closed subset $A$ is invariant under $T$ then $A=X$. It follows that $A=X$. Since the natural homomorphism $H^{n}(X) \rightarrow H^{n}(B)$ is onto, by the preceding argument, we have $H^{n}(B)$ $=0$. The theorem is proved.

We have the following corollary immediately.

CoRollaRy 1. Let $(X, T, \pi)$ be a transformation group, where $X$ is a compact Hausdorff space and $T$ is a connected topological group. Let $\operatorname{cd}(X) \leqq n$. If there is a proper subset $A$ of $X$ such that $H^{n}(A) \neq 0$ then $(X, T, \pi)$ is not a minimal set.

It is trivial that there is no minimal set $(X, R, \pi)$ with $\operatorname{cd}(X)=0$ under continuous flow, except $X$ contains a single point only. However, it is not the case if the transformation group is a discrete flow. In fact, for example, every universal minimal set $(X, Z, \pi)$ under discrete flow (see [6]) is of $\operatorname{cd}(X)=0$, because $X$, in this case, is totally disconnected. (See [4].)

The following corollary is an open question raised by Professor W. H. Gottschalk in an address. (See [8].) The answer to this question is negative.

CoROllaRY 2. The universal curve, $M$, of Menger and the universal curve, $S$, of Sierpinski both can not be minimal under any connected topological group.

Proof. We know $\operatorname{cd}(M)=1$ and $\operatorname{cd}(S)=1$ and the fact that the circle $S^{\prime}$ can be topologically embedded into both $M$ and $S$. We may consider $S^{\prime}$ is a proper closed subset of $M$ and $S$ respectively. From the fact $H^{1}\left(S^{\prime}\right) \neq 0$ and Corollary 1 , this corollary is proved.

Remark 2. Professor R. D. Anderson proved that $M$ is a minimal set under a certain discrete flow, (see [1]), and Professor.W. H. Gottschalk proved that $S$ is not a minimal set under any discrete flow, (see [9]).

Corollary 3. Let $(X, T, \pi)$ be a transformation group where $X$ is a compact Hausdorff space and $T$ is a connected topological group. Let 
$\operatorname{cd}(X) \leqq n$ and $x \in X$. If there exists a proper, closed subset $A$ of $\mathrm{Cl}(T x)$ such that $H^{n}(A) \neq 0$ then $x$ is not an almost periodic point.

Proof. This is a consequence of Corollary 1 and the facts that if $x$ is an almost periodic point (e.g. see $[7 ; 12])$ then $(\mathrm{Cl}(T x), T, \pi)$ is minimal and $\operatorname{cd}(\mathrm{Cl}(T x)) \leqq \mathrm{cd}(X)$. (See [6].)

Corollary 4 Let $(X, Z, \pi)$ be a discrete flow where $X$ is a compact Hausdorff space with $\operatorname{cd}(X) \leqq n$. Let $t$ be the generator of $Z$. If $(X, Z, \pi)$ is minimal and if there exists a nonzero integer $r$ such that $t^{r}$ is homotopic to identity then $H^{n}(B)=0$ for every proper, closed subset $B$ of $X$.

Proof. Let $Z^{\prime}$ be the subgroup generated by $t^{r}$ of $Z$. Then $\left(X, Z^{\prime}, \pi\right)$ is a minimal set. Complete the proof by the Remark 1 after Lemma 1.

The following result, due to A. A. Markoff, is well-known. "Let $(X, T, \pi)$ be a transformation group, where $X$ is a compact metrizable space of inductive dimension $n$ (we denote this by $\operatorname{ind}(X)=n$ ) and it contains more than one point and $T$ is a connected topological group. If $X$ is minimal then it can not be disconnected by a closed subset of inductive dimension $\leqq n-2$." (E.g. see $[7 ; 11 ; 12]$.) We generalize this result as follows:

TheOREM 2. Let $(X, T, \pi)$ be a transformation group, where $X$ is compact Hausdorff space and it contains more than one point and $T$ is a connected topological group. If $X$ is minimal and if there exists a closed set $D$ of $X$ such that $H^{n}(X, D) \neq 0$ then it can not be disconnected by a closed subset of cohomology dimension $\leqq n-2$ and for every $x \in X$, there exists an open neighborhood $V_{x}$ of $x$ such that $H^{n}\left(X, X-V_{x}\right) \neq 0$.

Proof. Let $\zeta \in H^{n}(X, D), \zeta \neq 0$ and let $A$ be one of its floors. Then it is easy to see that for every $t \in T, t A$ is a floor of $\left(t^{*}\right)^{-1} \zeta \in H^{n}(X, t D)$, $\left(t^{*}\right)^{-1} \zeta \neq 0$, where the isomorphism $t^{*}: H^{n}(X, t D) \rightarrow H^{n}(X, D)$ is induced by $t$. Suppose the $X$ can be disconnected by a closed subset of cohomology dimension $\leqq n-2$, it is not hard to see that there exist two closed proper sets $B_{1}$ and $B_{2}$ such that $X=B_{1} \cup B_{2}$ and $B_{1} \cup B_{2}=C$ where $\operatorname{cd}(C) \leqq n-2$. Consider the following Mayer-Vietoris sequence,

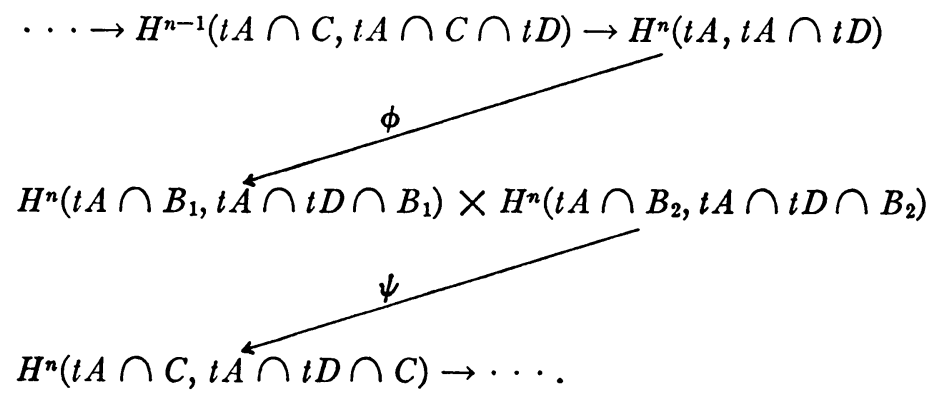


Since $\operatorname{cd}(C) \leqq n-2$, we have $\operatorname{cd}(t A \cap C, t A \cap t D \cap C) \leqq n-2$. Consequently $H^{n}(t A \cap C, t A \cap t D \cap C)=H^{n-1}(t A \cap C, t A \cap t D \cap C)=0$. It follows that $\phi$ is an isomorphism onto. Since $t A$ is a floor of $\left(t^{*}\right)^{-1} \zeta$ $\in H^{n}(X, t D),\left(t^{*}\right)^{-1} \zeta \neq 0, \phi$ can not be isomorphic unless $t A \subset B_{1}$, or $t A \subset B_{2}$. However, $t A$ can not be contained in both $B_{1}$ and $B_{2}$, otherwise $t A \subset B_{1} \cap B_{2}=C, \operatorname{cd}(t A) \leqq \operatorname{cd}(C) \leqq n-2$ and $H^{n}(t A, t A \cap t D)$ $=0$ or $\left.\left(t^{*}\right)^{-1}\right\}=0$. Let $T_{1}=\left\{t \mid t A \subset B_{1}, t \in T\right\}$ and $T_{2}=\left\{t \mid t A \subset B_{2}, t \in T\right\}$. It is clear that $T_{1}$ and $T_{2}$ are closed and $T_{1} \cap T_{2}=\phi$ and $T_{1} \cup T_{2}=T$. We know, however, $T$ is connected. This implies either $T_{1}$ or $T_{2}$ is empty. Say $T_{2}$ is empty, we have $t A \subset B_{1}$ for all $t \in T$. Since $X$ is minimal, we have $\operatorname{Cl}(T A)=X=B_{1}$, it follows that $B_{2}$ is empty. The first part of the theorem is proved.

For $x \in X-D$, we choose $V_{x}=X-D$; then $D=X-V_{x}$. We have $H^{n}\left(X, X-V_{x}\right)=H^{n}(X, D) \neq 0$. For $x \notin X-D$, we have $x \in D$. Then there exists $t_{x} \in T$ such that $x \notin t_{x} D$. Otherwise, $t x \in D$ for all $t \in T$. Consequently $\mathrm{Cl}(T x)=X \subset D$ or $X=D$. It follows that $H^{n}(X, D)$ $=H^{n}(X, X)=0$. A contradiction! Therefore, for $x \in D$, we choose $V_{x}=X-t_{x} D$. Then $H^{n}\left(X, X-V_{x}\right)=H^{n}\left(X, t_{x} D\right) \cong H^{n}(X, D) \neq 0$.

REMARK 3. The Theorem 2 is still valid, if we change the cohomology dimension in the statement to be covering dimension or inductive dimension. This is easily seen from the proof of Theorem 2.

CoRollary 5. Let $(X, T, \pi)$ be a transformation group, where $X$ is compact Hausdorff space of $\operatorname{cd}(X)=n$ (covering dimension or inductive dimension of $X=n$ denote by $\operatorname{cov}(X)=n$ or $\operatorname{ind}(X)=n$ ) and it contains more than one point and $T$ is connected. If $X$ is minimal then it can not be disconnected by a closed subset of cohomology dimension (covering dimension, or inductive dimension) $\leqq n-2$, and for every $x \in X$, there exists an open neighborhood $V_{x}$ of $x$ such that $H^{n}\left(X, X-V_{x}\right) \neq 0$.

Proof. Since $\operatorname{cd}(X)=n$, there exists a closed set $D$ in $X$ such that $H^{n}(X, D) \neq 0$. Complete the proof by Theorem 2 and Remark 3 , and well-known inequalities: $\operatorname{cd}(X) \leqq \operatorname{ind}(X)$ and $\operatorname{cd}(X) \leqq \operatorname{cov}(X)$.

REMARK 4. Notice that the metrizability of $X$ is not assumed in Theorem 2 as well as Corollary 5.

Corollary 6. (Added in proof). Let $(X, T, \pi)$ be a transformation group where $X$ is a compact Hausdorff space of $\operatorname{ind}(X)=n$ and $T$ is connected. If $X$ is minimal then every point of $X$ has the same dimension $n$.

\section{REFERENCES}

1. R. D. Anderson, Minimal sets under flows on tori and derived spaces, Notices Amer. Math. Soc. 5 (1958), 843. 
2. G. D. Birkhoff, Dynamic systems, Amer. Math. Soc. Colloq. Publ. Vol. 9, Amer. Math. Soc., New York, 1927.

3. A. Borel et al., Seminar on transformation groups, Princeton Univ. Press, Princeton, N. J., 1960.

4. H. Cohen, $A$ cohomological definition of dimension for locally compact Hausdorff spaces, Duke Math. J. 21 (1954), 209-224.

5. S. Eilenberg and N. E. Steenrod, Foundations of algebraic topology, Princeton Univ. Press, Princeton, N. J., 1952.

6. R. Ellis, On universal minimal sets, Proc. Amer. Math. Soc. 11 (1960), 540-543.

7. W. H. Gottschalk and G. A. Hedlund, Topological dynamics, Amer. Math. Soc. Colloq. Publ. Vol. 36, Amer. Math. Soc., Providence, R. I., 1955.

8. W. H. Gottschalk, Minimal sets, Bull. Amer. Math. Soc. 64 (1958), 336-351.

9. - The universal curve of Sierpinski is not a minimal set, Notices Amer. Math. Soc. 6 (1959), 257.

10. W. Hurewicz and H. Wallman, Dimension theory, Princeton Univ. Press, Princeton, N. J., 1948.

11. A. A. Markov, Sur une propriêté generale des ensembles minimeux de M. Birkhoff, C. R. Acad. Sci. Paris, 193 (1931), 823-825.

12. V. V. Nemyckil and V. V. Stepanov, Qualitative theory of differential equations, Princeton Univ. Press, Princeton, N. J., 1960.

13. E. Spanier, Cohomology theory for general spaces, Ann. of Math. (2) 49 (1948), 407-427.

NORTHWESTERN UNIVERSITY AND

University of British Columbia 